Available online at www.eccomasproceedia.org

Eccomas Proceedia COMPDYN (2021) 3605-3616

ECCOMAS

Proceedia
COMPDYN 2021

$8^{\text {th }}$ ECCOMAS Thematic Conference on Computational Methods in Structural Dynamics and Earthquake Engineering

M. Papadrakakis, M. Fragiadakis (eds.) Streamed from Athens, Greece, 28 - 30 June 2021

\title{
ELASTODYNAMIC ANALYSIS OF LATERALLY LOADED PILES: MODIFICATIONS TO A SIMPLIFIED ENERGY APPROACH
}

\author{
Jamie J. Crispin ${ }^{1}$, and George E. Mylonakis ${ }^{123}$ \\ ${ }^{1}$ Department of Civil Engineering \\ University of Bristol, Bristol, UK \\ j.crispin@bristol.ac.uk,g.mylonakis@bristol.ac.uk \\ ${ }^{2}$ Department of Civil Engineering \\ University of California at Los Angeles, Los Angeles, CA, USA \\ ${ }^{3}$ Department of Civil and Infrastructure Engineering \\ Khalifa University, Abu Dhabi, UAE
}

\begin{abstract}
A simple, yet powerful analytical model for determining the dynamic response of laterally loaded piles has recently been proposed in a series of papers by Karatzia and Mylonakis. The method is essentially a finite-element formulation, based on the evaluation of a set of energy integrals to establish the dynamic stiffness and damping matrices at the pile head. The key ideas/assumptions behind the method are: (1) the soil around the pile is replaced by a bed of dynamic Winkler springs and dashpots accounting for soil stiffness and energy dissipation; (2) a shape function for pile deflection is employed along the whole pile length in each vibration mode; (3) the associated integrals can be solved in closed form. Solutions have been obtained for different soil profiles which provided realistic predictions of pile response to flexural loads. An implicit assumption of the method lies in the use of real-valued shape functions. While such functions greatly simplify the analysis by separating real and imaginary parts (thus leading exclusively to real-valued integrals), they have the disadvantage of ignoring the phase differences between pile movements at different depths.

This paper recognises that this simplification can lead to inaccurate results for inhomogeneous soil profiles, especially at high frequencies where phase differences among different points along the pile are significant. To overcome the problem, the possibility of using complex-valued shape functions, analogous to those employed in spectral finite-element methods is explored. It is shown, through comparison with rigorous numerical solutions (including FEM and BEM), that use of complex-valued shape functions improves the predictive power of the method.
\end{abstract}

Keywords: Pile, Winkler, Finite Elements, Spectral Elements, Shape Function, Virtual Work. 


\section{INTRODUCTION}

Winkler based models are well established for use in elastodynamic analysis of laterally loaded piles. Early work provided analytical expressions for the spring and dashpot coefficients based on a horizontal soil "slice" model $[1,2,3]$. Several subsequent studies provided solutions for pile stiffness and damping in homogeneous and inhomogeneous media, as well as kinematic response coefficients to seismic excitation $[4,5,6,8,9,10,11,12,15,16,25,26,27]$. A difficulty in employing such solutions in design lies in the inability to derive exact solutions for inhomogeneous media in the dynamic regime. An alternative approach is explored in this paper, by means of an energy formulation analogous to those used in finite-element methods, based on single shape function for the whole pile length. The method was introduced in pile dynamics by Dobry and Roesset [6] and later extended in a series of papers by Mylonakis and co-workers $[8,9,10,11,16,26]$. The main assumptions of the method are: (1) the soil around and under the pile can be replaced by a bed of dynamic springs and dashpots accounting for soil stiffness and pile-soil energy dissipation (Winkler approximation); (2) a single shape function can be employed for the deflection of the whole pile for each vibration mode (Rayleigh approximation); (3) a virtual work formulation can be employed in which the associated one-dimensional integrals can be evaluated in closed form. In this way, the three-dimensional elastodynamic problem reduces to a handful of one-dimensional integrals which can be evaluated in closed form for different inhomogeneous soil media. The solutions have been shown to provide realistic predictions of pile response to flexural loads.

A simplification associated with the above method lies in the use of real-valued shape functions for pile deflection. While such functions greatly simplify the complex arithmetic by separating real and imaginary parts leading to exclusively real-valued integrals, they have the disadvantage of ignoring the phase differences between pile displacements at different depths. This paper recognises that this simplification can lead to inaccurate results for inhomogeneous soil profiles, especially at high frequencies where the phase differences between displacements at different elevations along the pile are significant. To overcome the problem, the possibility of using complex-valued shape functions, analogous to those employed in spectral finite element methods, is explored in this paper.

\section{PROBLEM DEFINITION}

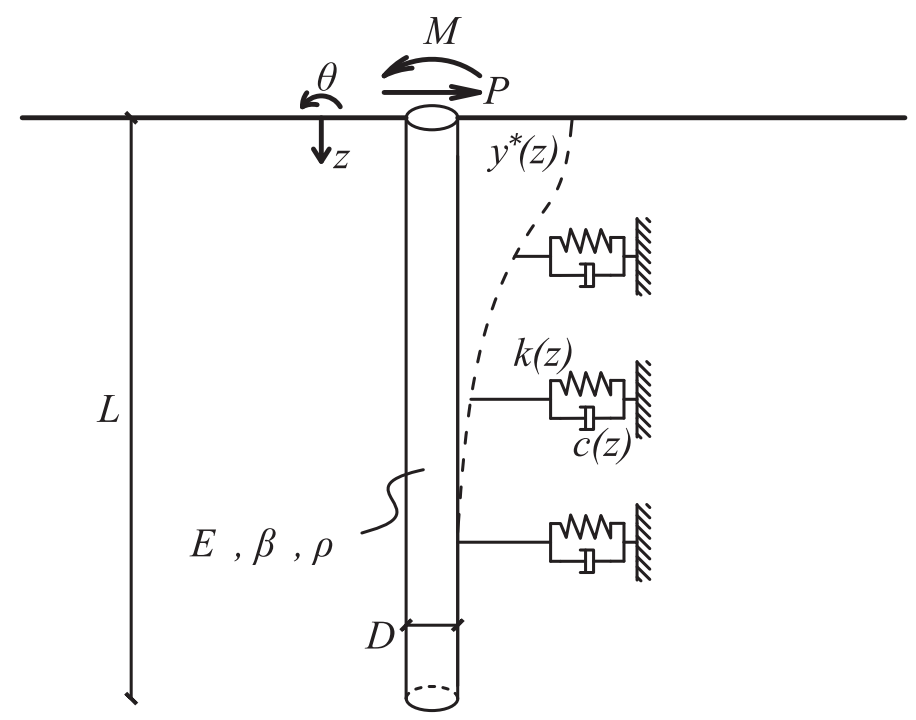

Figure 1: The dynamic Winkler model for a laterally loaded pile. 
The dynamic Winkler model under consideration is shown in Figure 1. The pile is modelled as elastic Euler-Bernoulli beam with length, $L$, diameter, $D$, cross sectional area, $A$, second moment of area, $I$, mass density, $\rho_{p}$, elastic stiffness, $E_{p}$ and hysteretic material damping coefficient, $\beta_{p}$. The last two terms can be expressed as a complex viscoelastic stiffness, $E_{p}^{*}=$ $E_{p}\left(1+2 i \beta_{p}\right)$.

A harmonic head load, $P$, and/or (anticlockwise positive) moment, $M$, are applied at an angular frequency, $\omega$, to the pile head. This is resisted by distributed Winkler springs and dashpots with coefficients $k(z)$ and $c(z)$, respectively, which are often expressed as a complex-valued stiffness, $k^{*}=k+i \omega c$. The resulting pile displacement, $y^{*}(z)$, is also complex-valued, indicating a depth-varying phase difference from the applied load.

The governing equation describing the problem is obtained from the well-known expression for a beam under a distributed load [4]:

$$
E_{p}^{*} I y^{* \prime \prime \prime \prime}(z)+\left[k(z)+i \omega c(z)-\rho_{p} A \omega^{2}\right] y^{*}(z)=0
$$

where $y^{* \prime \prime \prime \prime}(z)$ indicates the fourth derivative of $y^{*}(z)$ with respect to depth, $z$.

Of most interest is the response at the pile head, which can be described using a complexvalued stiffness matrix, $\boldsymbol{K}^{*}$, defined as:

$$
\boldsymbol{P}=\left[\begin{array}{l}
P \\
M
\end{array}\right]=\boldsymbol{K}^{*} \boldsymbol{u}^{*}=\left[\begin{array}{ll}
K_{11}^{*} & K_{12}^{*} \\
K_{21}^{*} & K_{22}^{*}
\end{array}\right]\left[\begin{array}{l}
y_{0}^{*} \\
\theta_{0}^{*}
\end{array}\right]
$$

where $\boldsymbol{P}$ and $\boldsymbol{u}^{*}$ are the load and displacement vectors at the pile head, respectively, in which $y_{0}^{*}$ is the pile head displacement and $\theta_{0}^{*}$ is the pile head rotation (anti-clockwise positive). The components of the stiffness matrix $K_{11}^{*}, K_{22}^{*}$ and $K_{12}^{*}\left(=K_{21}^{*}\right)$ are the swaying, rocking and cross swaying-rocking complex stiffnesses, respectively. Due to the linear-elastic nature of the problem, the matrix is symmetric $\left(K_{12}^{*}=K_{21}^{*}\right)$. In addition, with the sign convention chosen, all the stiffness terms are positive at low frequency.

For convenience, it is common to separate the complex stiffness components into real-valued spring and dashpot coefficients, $K_{i j}$ and $C_{i j}$ :

$$
\begin{gathered}
K_{i j}=\mathcal{R}\left[K_{i j}^{*}\right] \\
C_{i j}=\frac{1}{\omega} \mathcal{J}\left[K_{i j}^{*}\right]
\end{gathered}
$$

where $\mathcal{R}[]$ and $\mathcal{J}[]$ indicate the real and imaginary components, respectively.

\section{CLOSED FORM SOLUTION}

For the homogeneous case, where $k(z)=k$ and $c(z)=c$, a complex wavenumber, $\lambda^{*}$ can be introduced such that equation 1 reduces to:

$$
\begin{gathered}
y^{* \prime \prime \prime \prime}(z)+4 \lambda^{* 4} y^{*}(z)=0 \\
\lambda^{* 4}=\left(k+i \omega c-\rho_{p} A_{p} \omega^{2}\right) /\left(4 E_{p}^{*} I\right)
\end{gathered}
$$

This expression matches the well-known governing equation for the static Winkler model, with some terms substituted for complex-valued dynamic terms. Therefore, the static solution can be employed with the corresponding substitutions. For a long pile (longer than the active length, discussed later), the head stiffness matrix is given by [4]:

$$
\boldsymbol{K}^{*}=\left[\begin{array}{cc}
4 E_{p}^{*} I \lambda^{* 3} & 2 E_{p}^{*} I \lambda^{* 2} \\
2 E_{p}^{*} I \lambda^{* 2} & 2 E_{p}^{*} I \lambda^{*}
\end{array}\right]
$$


This expression is simple and easy to implement. However, no closed-form solutions to equation 1 are known to the authors for any inhomogeneous stiffness profiles encountered in practice. In addition, although a closed-form solution is available for piles in layered soil (in the form of successive substitution into a formula for each layer), this results in an unwieldy expression. Instead, an approximate energy solution can be employed.

\section{ENERGY SOLUTION}

Consider a pile in equilibrium subjected to an actual deflection $y^{*}(z)$ under the influence of a set of external loads at the pile head and tip; the work done by a small virtual displacement, $y_{v}(z)$, on the system can be obtained by integrating equation 1 :

$$
E_{p}^{*} I \int_{0}^{L} y^{* \prime \prime \prime \prime}(z) y_{v}(z) d z+\int_{0}^{L}\left[k^{*}(z)-\rho_{p} A \omega^{2}\right] y^{*}(z) y_{v}(z) d z=0
$$

This is known as the strong form of the equation; the desired weak form can then be obtained using integration by parts and the Euler-Bernoulli beam relationships at the pile head. Note that for long piles, the base response (and therefore upper integration limit) has negligible effect on the results. Therefore, these can be expressed as improper integrals:

$$
P y_{v}(0)+M y_{v}^{\prime}(0)=E_{p}^{*} I \int_{0}^{\infty} y^{* \prime \prime}(z) y_{v}^{\prime \prime}(z) d z+\int_{0}^{\infty}\left[k^{*}(z)-\rho_{p} A \omega^{2}\right] y^{*}(z) y_{v}(z) d z=0(7)
$$

The stiffness matrix coefficients can then be obtained by dividing equation 7 by the displacement and virtual displacement at the pile head:

$$
K_{i j}^{*}=E_{p}^{*} I \int_{0}^{\infty} \chi_{i}^{\prime \prime}(z) \chi_{j}^{\prime \prime}(z) d z+\int_{0}^{\infty}\left[k^{*}(z)-\rho_{p} A_{p} \omega^{2}\right] \chi_{i}(z) \chi_{j}(z) d z
$$

where $\chi_{i}(z)$ and $\chi_{j}(z)$ are dimensionless, unitary shape functions describing the displacement and virtual displacement variation with depth, respectively.

A similar approach has previously been employed by $[5,6,7]$ to obtain pile head dashpot coefficients for the swaying mode $\left(C_{11}\right)$. In the form shown here (for complex pile head stiffness), the method was first employed by [8]. Analytical expressions for layered soil were first included in [4, 8], a linear Winkler stiffness profile in [9], a parabolic Winkler stiffness profiles in [10] and a power-law and an exponential Winkler stiffness profiles in [11].

These solutions have used the following shape functions from the solution for homogeneous Winkler stiffness profiles [8]:

$$
\begin{gathered}
\chi_{1}(z)=e^{-\mu z}[\sin (\mu z)+\cos (\mu z)] \\
\chi_{2}(z)=\frac{e^{-\mu z}}{\mu} \sin (\mu z)
\end{gathered}
$$

where $\mu$ is a real-valued average wavenumber analogous to $\lambda^{*}$ for the static case $(\omega=0)$, the inverse of which can be used as a characteristic pile wavelength [12]. For homogeneous profiles, $\mu$ is set equal to $\lambda^{*}$ in equation 4 with $\omega=0$ (the static case), calculating $\mu$ for other Winkler stiffness profiles is discussed later. Note that the component $\boldsymbol{K}^{*}\left(K_{i j}^{*}\right)$ obtained in equation 8 corresponds to the subscripts of the shape function chosen for $\chi_{i}$ and $\chi_{j}$ [e.g. $K_{i j}^{*}$ is obtained using $\chi_{i}=\chi_{1}$ and $\left.\chi_{j}=\chi_{2}\right]$.

These real-valued shape functions neglect the difference in phase of the response down the pile. However, they have the significant advantage that equation 8 can easily be separated into real-valued pile head stiffness and dashpot coefficients using equation 3 . The pile head stiffness terms are given by $[10,11]$ :

$$
K_{i j}=E_{p} I \int_{0}^{\infty} \chi_{i}^{\prime \prime}(z) \chi_{j}^{\prime \prime}(z) d z+\int_{0}^{\infty} k(z) \chi_{i}(z) \chi_{j}(z) d z-\rho_{p} A_{p} \omega^{2} \int_{0}^{\infty} \chi_{i}(z) \chi_{j}(z) d z
$$


The first two terms (the contribution of the pile flexural stiffness and soil stiffness, respectively) make up the static pile head stiffness and are independent of $\omega$. The last term is the contribution of the pile inertia and is often neglected in the analysis as it is typically a minor component [11].

The pile head damping terms are given by $[10,11]$ :

$$
C_{i j}=\frac{2 \beta_{p} E_{p} I}{\omega} \int_{0}^{\infty} \chi_{i}^{\prime \prime}(z) \chi_{j}^{\prime \prime}(z) d z+\frac{2 \beta_{s}}{\omega} \int_{0}^{\infty} k(z) \chi_{i}(z) \chi_{j}(z) d z+\int_{0}^{\infty} c_{r}(z) \chi_{i}(z) \chi_{j}(z) d z(11)
$$

where the Winkler dashpot coefficient, $c(z)$, is split into the (hysteretic) material and radiation components, $c_{h}$ and $c_{r}$, respectively, and $\beta_{s}$ is the soil hysteretic material damping coefficient. The three terms of equation 11 correspond to the contributions of the pile material damping, soil material damping and radiation damping, respectively.

The simplification in equations 10 and 11 allows the pile head response to be evaluated from a few simple, real-valued, integrals. If the inertial term in equation 10 is neglected (note that in this paper it is not), only three unique integrals need to be evaluated. The first two are common to both equations, the first of which is also independent of the Winkler stiffness profile encountered. Analytical solutions for these integrals in closed-form are collated by [11] for all of the aforementioned Winkler stiffness profiles and a simple solution is included in [13] based on the result for homogeneous profiles.

\section{APPLYING THESE SOLUTIONS: HOMOGENEOUS SOIL}

In order to apply these solutions for homogeneous soil, only the Winkler spring and dashpot coefficients must be determined.

\section{$5.1 \quad$ Selection of $\boldsymbol{k}^{*}$}

Various solutions are available for the selection of $k^{*}$, including analytical approximations and empirical functions fitted against numerical continuum solutions. The Winkler spring stiffness, $k$, is often assumed to be proportional to the elastic and/or shear moduli, $E_{s}$ and $G_{s}$, respectively while the radiation dashpot coefficient, $c_{r}$, is often assumed to be proportional to the soil shear wave velocity, $V_{s}=\sqrt{G_{S} / \rho_{s}}$, where $\rho_{s}$ is the soil mass density. A number of available expressions are tabulated in $[14,15,16]$. In this paper, three different approaches are considered.

The first approach is the horizontal slice model employed by $[1,2,3]$. The soil is modelled as discrete, infinite, horizontal slices under zero vertical normal strain. The equivalent spring and dashpot at the pile-soil interface is then solved in rigorously in closed-form. In [3] the hysteretic material damping is incorporated using complex-valued moduli from the correspondence principle of viscoelasticity [17]:

$$
\begin{aligned}
G_{S} & \rightarrow G_{s}^{*}=G_{s}\left(1+2 i \beta_{s}\right) \\
E_{s} & \rightarrow E_{s}^{*}=E_{s}\left(1+2 i \beta_{s}\right) \\
V_{s} & \rightarrow V_{s}^{*}=V_{s} \sqrt{1+2 i \beta_{s}}
\end{aligned}
$$

This results in the following expression for the complex-valued stiffness, $k^{*}$ :

$$
\begin{gathered}
k^{*}=\pi G_{s}^{*} s^{2} \frac{4 K_{1}(q) K_{1}(s)+s K_{1}(q) K_{0}(s)+q K_{0}(q) K_{1}(s)}{s K_{1}(q) K_{0}(s)+q K_{0}(q) K_{1}(s)+q s K_{0}(q) K_{0}(s)} \\
s=\frac{i \omega D}{2 V_{s} \sqrt{1+2 i \beta_{s}}} \\
q=s \sqrt{\frac{1-2 v_{s}}{2\left(1-v_{s}\right)}}
\end{gathered}
$$


where $K_{v}()$ is the modified Bessel function of the second kind of order $v$. While the analytic nature of this expression is appealing, it does yield zero static stiffness at low frequencies. In addition, due to the Bessel functions, this expression cannot be conveniently separated into real and imaginary parts in closed form.

As an alternative, [18] provided the simple expression in equation 14, which was fitted against numerical finite element results for a pile embedded in a homogeneous half space. The hysteretic damping term is treated approximately by only considering the effect on the real part of the stiffness.

$$
\begin{gathered}
k=1.2 E_{s} \\
c_{r}=5 \rho_{s} V_{s} D \\
c_{h}=2 \beta_{s} \frac{k}{\omega}
\end{gathered}
$$

Finally, the model employed by [11] is considered. This model uses the [19] approach previously applied to this problem by [20,21], which yields an improved version of the horizontal slice model that gives finite stiffness at low frequency. However, unlike in the previous models, this is only used employed for the real-valued coefficient, for which a simplified approximate expression is developed using the asymptotic behaviour of the Bessel functions. For the radiation damping coefficient, this model employs an improved version of the cone model introduced by [7]. Each horizontal slice is split into infinitesimal independent sectors through which both shear and compression waves propagate, resulting in a frequency dependant expression in terms of Bessel functions to which [11] fit the simple expression in equation 15. The hysteretic damping term is treated using the same approximation as in equation 14.

$$
\begin{gathered}
k=\frac{4 \pi G_{s} \eta_{u}^{2}}{\left(1+\eta_{u}^{2}\right)\left[\ln \left(4 / \alpha_{c}\right)-\gamma\right]+\ln \left(\eta_{u}\right)} \\
c_{r}=\pi \rho_{s} V_{s} D\left[\frac{1}{4}+\frac{4}{5} \sqrt{\frac{2}{1-v_{s}}}\right]\left(\frac{\omega D}{V_{s}}\right)^{-0.4} \\
c_{h}=2 \beta_{s} \frac{k}{\omega}
\end{gathered}
$$

where $\gamma(\approx 0.577)$ is the Euler-Mascheroni constant, $\eta_{u}=\sqrt{\left(2-v_{s}\right) /\left(1-v_{s}\right)}$ is a compressibility coefficient and $\alpha_{c}$ is a stiffness parameter (sometimes interpreted as a dimensionless cut-off frequency). Reference [11] provides simple power-law relationships between $\alpha_{c}$ and the pile-soil stiffness ratio, $E_{p} / E_{s}$, for different soil profiles and pile head fixity conditions. For fixed head piles in homogeneous soil $\alpha_{c}$ is given by:

$$
\alpha_{c}=1.227\left(\frac{E_{p}}{E_{s}}\right)^{-1 / 4}
$$

\subsection{Comparison of methods}

The performance of the closed-form and energy methods for each $k^{*}$ is shown in Figure 2, in comparison with numerical continuum results from $[22,23]$. The former used a boundary element method developed in [24] and the latter a finite element approach. The results are normalised by the well-known dimensionless frequency, $a_{0}=\omega D / V_{s}$.

In general, the analytical closed-form solution matches the continuum results reasonable well. The energy solution also performs very well at low frequencies, with a low discrepancy from the closed-form solution. However, as frequency increases, it tends to underestimate the pile head stiffness and overestimate the pile head damping coefficient. 

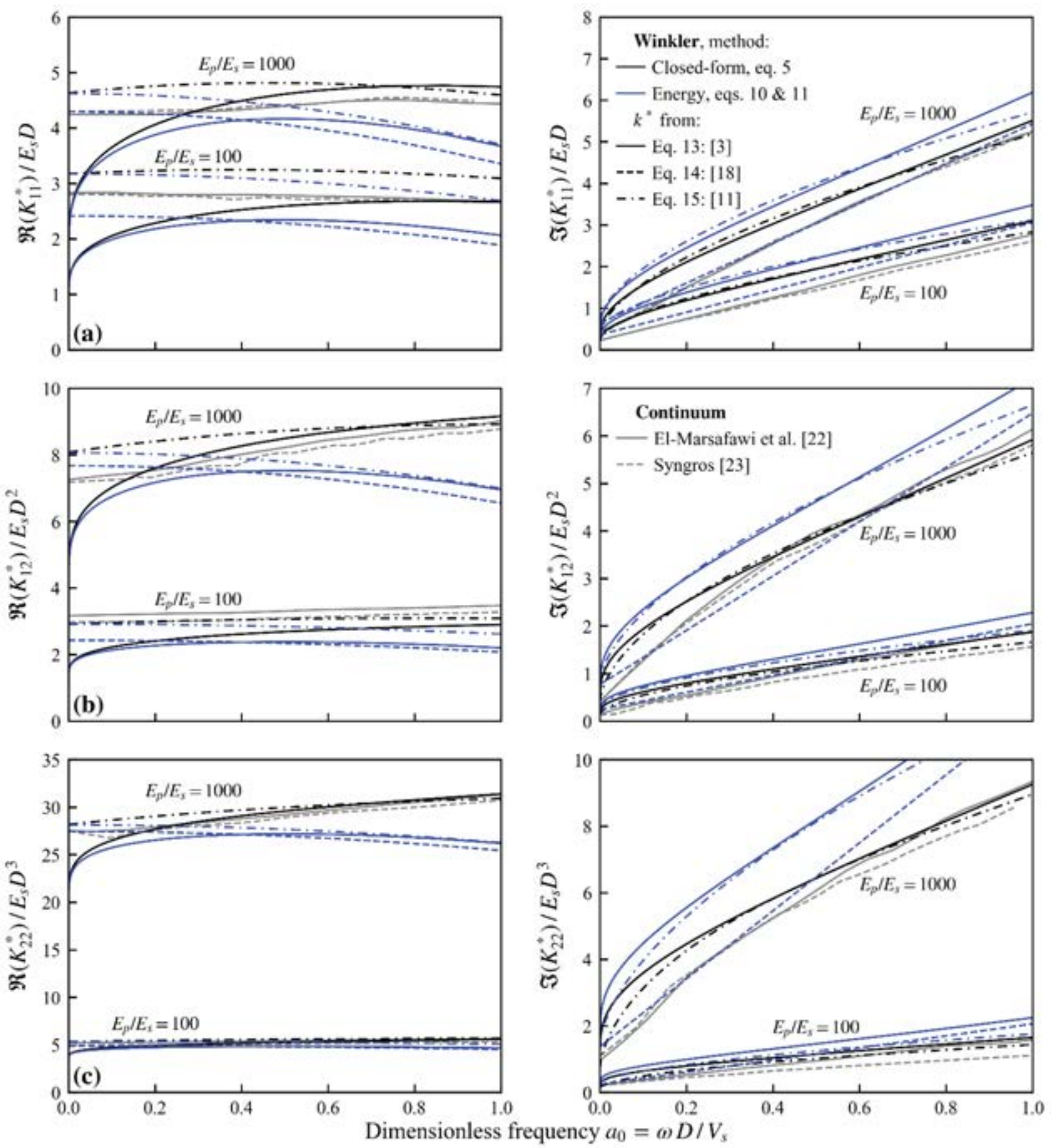

Figure 2: Normalised dynamic lateral head stiffness of piles embedded in a homogeneous half space; (a) $K_{11}^{*}$ (b) $K_{21}^{*}(\mathrm{c}) K_{22}^{*}\left(v_{s}=0.4, \beta_{s}=0.05, v_{p}=0.25, \beta_{p}=0.01, \rho_{p} / \rho_{s}=1.25\right)$ (numerical data from Syngros 2004).

\section{COMPLEX-VALUED SHAPE FUNCTIONS}

In order to get better predictions at higher-frequencies, [16] suggest using complex valued shape functions, $\chi_{i}^{*}$ and $\chi_{j}^{*}$ based on the dynamic (rather than static) response for the homogeneous case. Therefore, equation 8 can be rewritten in the from:

$$
K_{i j}^{*}=E_{p}^{*} I \int_{0}^{\infty} \chi_{i}^{* \prime \prime}(z) \chi_{j}^{* \prime \prime}(z) d z+\int_{0}^{\infty}\left[k^{*}(z)-\rho_{p} A_{p} \omega^{2}\right] \chi_{i}^{*}(z) \chi_{j}^{*}(z) d z
$$

where $\chi_{1}^{*}$ and/or $\chi_{2}^{*}$, given below, are substituted for $\chi_{i}^{*}$ and $\chi_{j}^{*}$ to get each of the complex stiffness terms as before. 


$$
\begin{gathered}
\chi_{1}^{*}(z)=e^{-\mu^{*} z}\left[\sin \left(\mu^{*} z\right)+\cos \left(\mu^{*} z\right)\right] \\
\chi_{2}^{*}(z)=\frac{e^{-\mu^{*} z}}{\mu^{*}} \sin \left(\mu^{*} z\right)
\end{gathered}
$$

$\mu^{*}$ is now directly analogous to $\lambda^{*}$; for homogeneous soil, if $\mu^{*}$ is set equal to $\lambda^{*}$, equation 17 reproduces the complex stiffness terms from the closed-form solution exactly, as the phase difference between the responses at different depths is accounted for correctly. However, for inhomogeneous soil, the integrals in equation 17 are now also complex-valued, and harder to compute than the solution with real-valued shape functions.

\section{APPLYING THESE SOLUTIONS: INHOMOGENEOUS SOIL}

In order to compare the performance of the two different assumptions (real-valued and complex-valued shape functions), an example problem involving a pile embedded in an inhomogeneous half-space is considered. In this example, soil stiffness varies according to a linear function of depth with zero surface stiffness. As with the homogeneous case, a method to calculate $k^{*}$ must be chosen. However, for inhomogeneous soil an appropriate method for selecting $\mu$ or $\mu^{*}$ must also be selected.

\subsection{Selection of $\boldsymbol{k}^{*}$}

Only two of the $k^{*}$ calculation approaches employed for homogeneous soil have been employed for this case, as the function fitted by [18] is specific to homogeneous soil. Equations 13 and 15 can be used as before, with $G_{s}$ and $V s$ simply referring to the values at each depth, resulting in an inhomogeneous Winkler stiffness profile. In addition, the modified expression for $\alpha_{c}$ for this case provided by [11] should be employed in place of equation 16 :

$$
\alpha_{c}=1.667\left(\frac{E_{p}}{E_{S D}}\right)^{-1 / 5}
$$

where $E_{S D}$ is the soil stiffness at a depth of one pile diameter.

However, the resulting functions describing the inhomogeneity in Winkler spring coefficients are not directly proportional to those describing the soil stiffness or shear wave velocity. Instead, for the solution described in equations 10 and 11, [11] suggests calculating $k^{*}$ only at a depth of one diameter. $k$ is then assumed proportional to the soil stiffness and $c_{r}$ to the soil shear wave velocity (the square root of soil stiffness).

\subsection{Selection of $\mu$}

For inhomogeneous soils, $\mu$ can be approximated as the average (static) $\lambda$ value over a certain depth. An appropriate depth, suggested by [8], is the pile active length, $L_{a}$, which can intuitively be interpreted as the depth over which the soil stiffness has an effect on the pile head response, resulting in the following expression for $\mu$ :

$$
\mu=\frac{1}{L_{a}} \int_{0}^{L_{a}}\left(\frac{k(z)}{4 E_{p} I}\right)^{1 / 4} d z
$$

A number of expressions are available in the literature for $L_{a}$, based on various different definitions. Particularly useful to application is the value of $\mu L_{a}=2.5$ suggested by [25], this allows equation 20 to be used iteratively to obtain $L_{a}$ and $\mu$ for any arbitrary Winkler stiffness profile. For the specific case of power-law Winkler stiffness profiles, this was solved analytically by [26]. For the linear profile considered here this results in the expression: 


$$
L_{a} / D=\left[\left(\frac{5}{4}\right)^{8} \frac{\pi E_{p}}{k_{D}}\right]^{1 / 5}
$$

where $k_{D}$ is the Winkler spring stiffness at one diameter depth.

\subsection{Selection of $\mu^{*}$}

$\mu^{*}$ can be calculated using a similar approach as $\mu$, taking the average (complex) $\lambda^{*}$ value over the active pile length:

$$
\begin{gathered}
\mu=\frac{1}{L_{a}} \int_{0}^{L_{a}} \lambda^{*}(z) d z \\
\lambda^{*}(z)=\left(\frac{k^{*}(z)-\rho_{p} A_{p} \omega^{2}}{4 E_{p}^{*} I}\right)^{1 / 4}
\end{gathered}
$$

As $\mu^{*} L_{a}$ is complex valued, the expression suggested by [25] cannot be employed. Instead, the simple expression fitted by [23] to finite element results in the same soil profile is used here:

$$
L_{a} / D=2.5\left(\frac{E_{p}}{E_{S D}}\right)^{1 / 5}
$$

\subsection{Comparison of methods}

The performance of the two different shape function assumptions is shown in Figure 3, in comparison with numerical continuum results from $[22,23]$. The results are normalised as before, using the shear wave velocity 20 diameters depth, $V_{s, 20 D}$, which was the pile length employed in the continuum solutions. An alternative normalisation, $a_{0 \mu}$, (shown in equation 24) was suggested in $[12,27]$ using $\mu$ directly. However, this was not suitable in this case due to the different $\mu$ definition for each solution.

$$
a_{0 \mu}=\frac{\omega}{\mu V_{s}(1 / \mu)}
$$

As for the homogeneous case, the energy method with real-valued shape functions matches the continuum results for low frequencies, but significantly underestimates the pile head stiffness and overestimates the pile head damping coefficient at higher frequencies. However, the complex-valued shape functions result in a solution much closer to the continuum results. Note that a rigid boundary was employed in both continuum solutions as perfect absorbing boundaries are not available for the inhomogeneous soil profile. This is not a limitation for the Winkler model, therefore the undulations of the impedance functions and cut-off frequency evident in the continuum results are not present.

\section{CONCLUSIONS}

An energy method for predicting the head response of laterally-loaded piles according the dynamic Winkler model has been investigated and extended. The performance of the approach with the commonly employed, real-valued shape functions has been compared with numerical continuum results, as well as the closed-form (Winkler) analytical solution for homogeneous soil and an alternative solution employing complex-valued shape functions in inhomogeneous soil. Three different methods for selecting the Winkler stiffness coefficients have been employed. The following conclusions can be drawn:

- The simplifying assumption of real-valued shape functions allows analytical expressions for the (real-valued) pile head stiffness and damping terms to be obtained, such as those provided in [11]. 

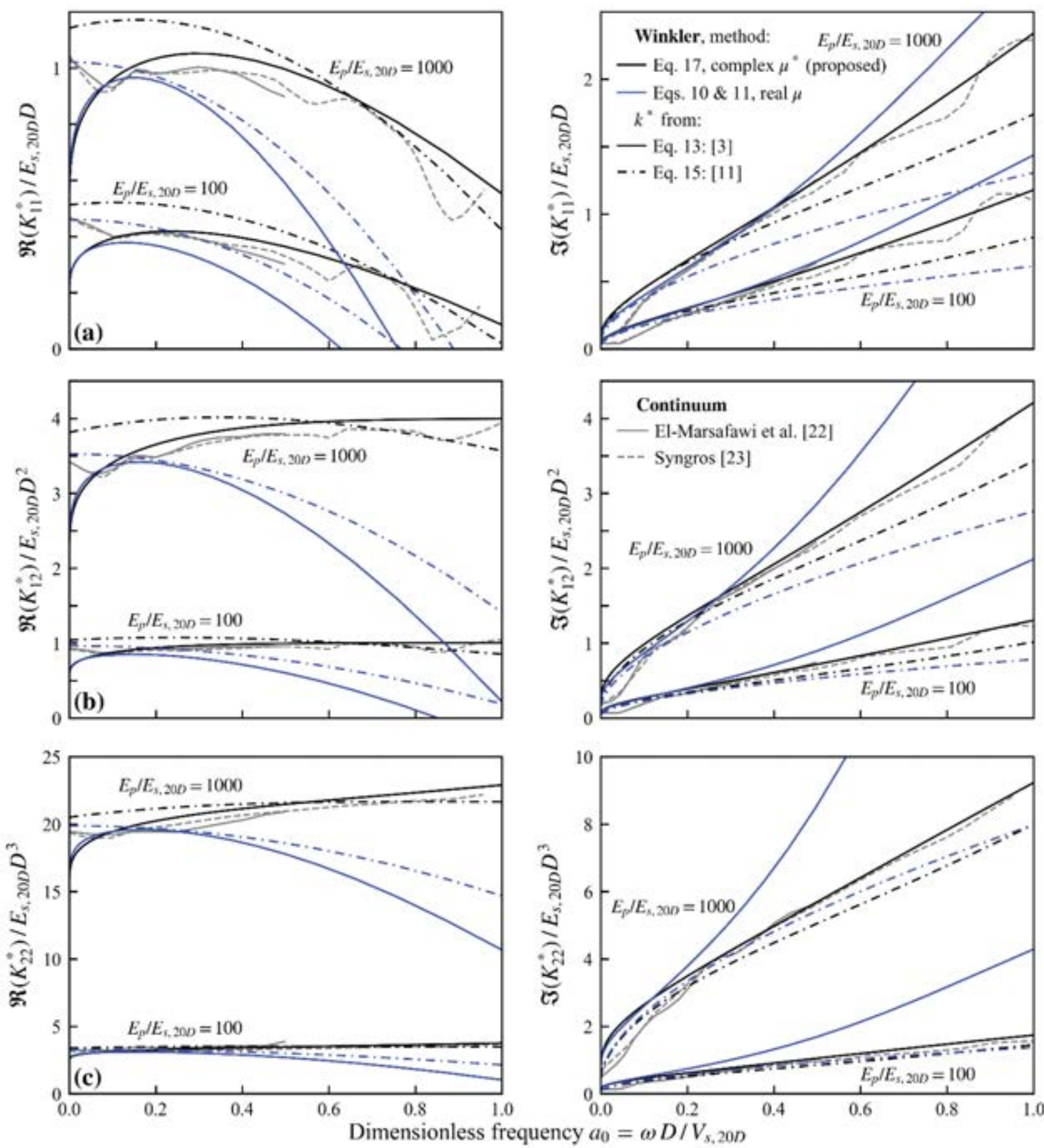

Figure 3: Normalised dynamic lateral head stiffness of piles embedded in an inhomogeneous soil with stiffness varying linearly with depth from a value of zero at the surface; (a) $K_{11}^{*}$ (b) $K_{21}^{*}$ (c) $K_{22}^{*}\left(v_{s}=0.4, \beta_{s}=\right.$ $\left.0.05, v_{p}=0.25, \beta_{p}=0.01, \rho_{p} / \rho_{s}=1.25\right)\left(L_{a}\right.$ and numerical data from Syngros 2004).

- At low frequencies, the energy method with real-valued shape functions gives similar results to the analytical, closed-from Winkler solution for homogeneous soil. However, as the frequency increases it underestimates pile head stiffness and overestimates pile head damping.

- The solution employing complex-valued shape functions reproduces the homogeneous analytical solution in closed-form.

- For the inhomogeneous soil considered, both real-valued and complex-valued shape functions provide similar results at low frequencies. However, at higher frequencies the real- 
valued shape functions result in underestimating pile head stiffness and overestimating pile head damping. This effect is more pronounced than for homogeneous soil.

- With the more rigorous complex-valued shape functions, the Winkler model performs well compared to the numerical continuum results in both the homogeneous and inhomogeneous soil profiles considered.

\section{REFERENCES}

[1] V.A. Baranov, On the calculation of excited vibrations of an embedded foundation (in Russian). Voprosy Dynamiki i Prochnoeti, (14), Polytechnic Institute, Riga, Latvia, 195 209, 1967.

[2] M. Novak, Dynamic stiffness and damping of piles. Canadian Geotechnical Journal, 11(4), 574-578, 1974.

[3] M. Novak, T. Nogami, F. Aboul-Ella, Dynamic soil reactions for plane strain case. Journal of Engineering Mechanics, ASCE, 104(4), 953-959, 1978.

[4] G. Mylonakis, G. Gazetas, Lateral vibration and internal forces of grouped piles in layered soil. Journal of Geotechnical and Geoenvironmental Engineering, ASCE, 125(1), 16-25, 1999.

[5] M.J. O'Rourke, R. Dobry, Spring and dash pot coefficients for machine foundation on piles. American Concrete Institute, Detroit, MI, USA. Report number SP-10, 177-198, 1978 .

[6] R. Dobry, E. Vicente, M.J. O'Rourke, J.M. Roesset, Horizontal stiffness and damping of single piles. Journal of Geotechnical Engineering, ASCE, 108(3), 439-459, 1982.

[7] G. Gazetas, R. Dobry, Simple radiation damping model for piles and footings. Journal of Geotechnical Engineering, ASCE, 110(6), 937-956, 1984.

[8] G. Mylonakis, Contributions to static and seismic analysis of piles and pile-supported bridge piers. Ph.D. thesis, State University of New York at Buffalo, 1995.

[9] G. Mylonakis, D. Roumbas, Lateral impedance of single piles in inhomogeneous soil. Proceedings of the 4th International Conference on Recent Advances in Geotechnical Earthquake Engineering and Soil Dynamics, paper 6.27, San Diego, CA, USA, March 26-31, 2001.

[10] X. Karatzia, G. Mylonakis, Horizontal response of piles in inhomogeneous soil: simple analysis. C. Soccodato, M. Maugeri eds. Proceedings of the 2nd International Conference on Performace-based Design in Earthquake Geotechnical Engineering, No 1117, Taormina, Italy, May 28-30 2012.

[11] X. Karatzia, G. Mylonakis, Horizontal stiffness and damping of piles in inhomogeneous soil. Journal of Geotechnical and Geoenvironmental Engineering, ASCE, 143(4), 04016113, 2017.

[12] E. Rovithis, G. Mylonakis, K. Pitilakis, Dynamic stiffness and kinematic response of single piles in inhomogeneous soil. Bulletin of Earthquake Engineering, 11(6), 1949-1972, 2013. 
[13] NEHRP, Soil-structure interaction for building structures. National Institute of Standards and Technology (NIST). Report number: GCR 12-917-21, 2012.

[14] M. Shadlou, S. Bhattacharya, Dynamic stiffness of pile in a layered elastic continuum. Géotechnique, 64(4), 303-319, 2014.

[15] G. Anoyatis, A. Lemnitzer, Dynamic pile impedances for laterally-loaded piles using improved Tajimi and Winkler formulations. Soil Dynamics and Earthquake Engineering, 92, 279-297, 2017.

[16] G.E. Mylonakis, J.J. Crispin, Simplified models for lateral static and dynamic analysis of pile foundations. A.M. Kaynia eds. Analysis of Pile Foundations Subject to Static and Dynamic Loading, CRC Press, in press, 2021.

[17] A.C. Pipkin, Lectures on viscoelastic theory. Springer, 1972.

[18] J.M. Roesset, Stiffness and damping coefficients of foundations. M. O’Neil, R. Dobry eds. Dynamic Response of Pile Foundations, ASCE, 1-29, 1980.

[19] V.Z. Vlasov, N.N. Leontiev, Beams, Plates and Shells on Elastic Foundations. Translated from Russian, Israel program for scientific translations, Washington, DC, USA. NIST No. N67-14238, 1966.

[20] G. Mylonakis, Elastodynamic model for large-diameter end-bearing shafts. Soils and Foundations, 41(3), 31-44, 2001.

[21] G. Anoyatis, G. Mylonakis, A. Lemnitzer, Soil reaction to lateral harmonic pile motion. Soil Dynamics and Earthquake Engineering, 87, 164-170, 2016.

[22] H. El-Marsafawi, A.M. Kaynia, M. Novak, Interaction factors and the superposition method for pile group dynamic analysis. Geotechnical Research Center, Department of Civil Engineering, University of Western Ontario, Canada, Report number GEOT-1-92, 1992.

[23] K. Syngros, Seismic response of piles and pile-supported bridge piers evaluated through case histories. Ph.D. thesis, The City College of the City University of New York, 2004.

[24] A.M. Kaynia, E. Kausel, Dynamic stiffness and seismic response of pile groups. Department of Civil Engineering, Massachusetts Institute of Technology, Cambridge, MA, USA, Research report no. R82-03, 1982.

[25] R. Di Laora, E. Rovithis, Kinematic bending of fixed-head piles in nonhomogeneous soil. Journal of Geotechnical and Geoenvironmental Engineering, ASCE, 141(4), 04014126, 2015.

[26] X. Karatzia, G. Mylonakis, Discussion of "Kinematic bending of fixed-head piles in nonhomogeneous soil" by Raffaele Di Laora and Emmanouil Rovithis. Journal of Geotechnical and Geoenvironmental Engineering, ASCE, 142(2), 07015042, 2016.

[27] G. Anoyatis, R. Di Laora, A. Mandolini, G. Mylonakis, Kinematic response of single piles for different boundary conditions: analytical solutions and normalization schemes, Soil Dynamics \& Earthquake Engineering, 44, 183-195, 2013. 